\title{
Synthesis and characterization of UV photocrosslinkable hydrogels with poly(N-vinyl- 2-pyrrolidone): Determination of the network mesh size distribution
}

Marizza, Paolo; Abrami, M.; Keller, Stephan Sylvest; Posocco, P.; Laurini, E.; Goswami, Kaustav; Skov, Anne Ladegaard; Boisen, Anja; Larobina, D.; Grassi, G.

Total number of authors:

11

Published in:

International Journal of Polymeric Materials and Polymeric Biomaterials

Link to article, DOI:

10.1080/00914037.2015.1129964

Publication date:

2016

Document Version

Peer reviewed version

Link back to DTU Orbit

Citation (APA):

Marizza, P., Abrami, M., Keller, S. S., Posocco, P., Laurini, E., Goswami, K., Skov, A. L., Boisen, A., Larobina, D., Grassi, G., \& Grassi, M. (2016). Synthesis and characterization of UV photocrosslinkable hydrogels with poly(N-vinyl-2-pyrrolidone): Determination of the network mesh size distribution. International Journal of Polymeric Materials and Polymeric Biomaterials, 65(10), 516-525.

https://doi.org/10.1080/00914037.2015.1129964

\section{General rights}

Copyright and moral rights for the publications made accessible in the public portal are retained by the authors and/or other copyright owners and it is a condition of accessing publications that users recognise and abide by the legal requirements associated with these rights.

- Users may download and print one copy of any publication from the public portal for the purpose of private study or research.

- You may not further distribute the material or use it for any profit-making activity or commercial gain

- You may freely distribute the URL identifying the publication in the public portal 


\title{
Characterisation of poly(n-vinyl-2-pyrrolidone) gels for the realisation of micro drug delivery systems
}

\author{
P. Marizza ${ }^{\text {a }}$, M. Abrami ${ }^{\text {b }}$, S. S. Keller ${ }^{\text {a }}$, P. Posocco ${ }^{\text {c }}$, E. Laurini ${ }^{\text {c }}$, K. Goswami ${ }^{\text {d }}$, A. L. Skov ${ }^{\text {d }}$, A. Boisen ${ }^{\text {a }}$, D. Larobina ${ }^{\text {e }}$, G. Grassi \\ ${ }^{\mathrm{b}}$ and M. Grassi ${ }^{\mathrm{c}}$
}

${ }^{a}$ Department of Micro- and Nanotechnology, Technical University of Denmark (DTU), Kongens Lyngby, Ørsteds Plads Bygning $345 \varnothing, 2800$ Kgs, Denmark.

${ }^{b}$ Department of Life Sciences, Cattinara Hospital, University of Trieste, Strada di Fiume 447, 34100 Trieste, Trieste, I-34127.

${ }^{c}$ Department of Engineering and Architecture, University of Trieste, via Valerio 6, Trieste, I-34127.

${ }^{d}$ Department of Chemical and Biochemical Engineering, Technical University of Denmark (DTU), Kongens Lyngby, Søltofts Plads 227, 2800 Kgs., Denmark.

${ }^{e}$ Department of Pharmacy, University of Copenhagen (KU), Universitetsparken 2, 2100 Copenhagen, Denmark

${ }^{f}$ National Research Council (CNR), Institute of Polymer Composites and Biomaterials, P.le Enrico Fermi 1, 80055 Portici Napoli Italy.

\begin{abstract}
Hydrogels of poly(n-vinyl-2-pyrrolidone) (PVP) were produced by UV irradiation of aqueous solutions of the polymer in presence of hydrogen peroxide, used as crosslinker. The mechanical and the nano-structural properties of the gels were characterized by a combination of experimental techniques including rheology, low field nuclear magnetic resonance spectroscopy (LFNMR) and small angle X-ray scattering (SAXS). Different irradiation doses as well as polymer and crosslinker concentrations were tested in the characterization. The study elucidates the relationship between different methods to estimate the mesh size of the gel polymeric network. Moreover, a novel correlation model was developed based on Chui and Scherer theories for the interpretation of LFNMR dataset of polymer solutions and networks.
\end{abstract}

\section{Introduction}

In recent years, special attention has been focused on hydrogels in the biomedical and pharmaceutical research. In fact, many features make this class of materials particularly suitable for the development of devices with drug delivery capabilities. ${ }^{1,2}$ In the last years, hydrogels found application in wound dressings ${ }^{3}$, injectable formulations ${ }^{4,5}$, dental medications ${ }^{6}$, implants ${ }^{7}$ and ophthalmic systems ${ }^{8}$. The principal characteristic of hydrogels is the large volume of water they absorb and retain which, together with intrinsic low cytotoxicity and high biocompatibility of the constituting polymers, place them among the most suitable materials resembling natural living tissues ${ }^{9,10}$. Moreover, hydrogels can be designed to serve as drug delivery device as they are able to release the active ingredient in a controlled manner

From a structural viewpoint, hydrogels are composed by hydrophilic polymeric three dimensional networks, where molecular chains are entangled or connected along limited areas or at punctual sites of the polymer backbone by physical junctions or chemical bonds. An important network feature is represented by crosslink density. Crosslink density determines the polymeric network mesh size, a characteristic that significantly affects the diffusion of a probe molecule. The correlation between release kinetics and the mesh size has been extensively studied ${ }^{11-14}$. Since the determination of the crosslink density is of paramount importance for the control of the 
drug release kinetics, the hydrogel preparation methods is a crucial step. Hydrogels can be prepared by crosslinking of both natural and fully synthetic hydrophilic polymers.

Exposure to high energy irradiation (gamma ray and electron beam) and UV light are among several different techniques adopted to produce hydrogels. The use of gamma radiation has been explored for PEG, PVP and agar based hydrogels ${ }^{15}$. The crosslinking upon exposure to UV radiation has recently attracted an increasing attention due to the simplicity of preparation method, and the implementation into micro-devices for drug delivery ${ }^{16-20}$. Several polymer derivatives were developed to implement photocrosslinking of active groups into polymer with oral delivery applications ${ }^{21-23}$. The crosslinking induced by UV radiation follows a radical mechanism ${ }^{24,25}$, activated by a photo-initiator, or crosslinker. The crosslinker attacks the polymer backbone generating macroradicals which react intermolecularly and thereby creating crosslinks. In many cases, the biocompatibility of photo-initiators is a critical issue as most of them are cytotoxic ${ }^{26}$. Therefore, the research of new crosslinking mechanisms represents a concrete need.

Poly(N-vinyl-2-pyrrolidone) (PVP) is a neutral and amphiphilic polymer which exhibits high solubility both in water and many organic solvents ${ }^{27}$ and is widely used as excipient for oral dosage forms ${ }^{28}$. PVP K30 and K90 can be crosslinked by exposure to UV radiation $^{29-31}$, giving a water insoluble gel material which swells in water. Photo-crosslinking of poly(vinylpyrrolidone) (PVP) was initially investigated by Rosiak and coworkers ${ }^{15}$ who used electron beam or gamma radiation to produce PVP hydrogel based-wound dressing. More recently, ultraviolet radiation (UV) was proposed by Lopérlogo et al. ${ }^{30}$ to crosslink aqueous solutions of PVP without the need for photo-initiators. The same authors added hydrogen peroxide $\left(\mathrm{H}_{2} \mathrm{O}_{2}\right)$ to the PVP solution as crosslinking agent increasing sensitively the gel fraction, that is the portion of polymer fixed in the network after irradiation, up to $90 \%{ }^{31}$. The structural and mechanical properties of these hydrogels were recently studied by D’Errico and coworkers ${ }^{32}$. In the present work, the attention is focused on the characterization of PVP photo-crosslinked hydrogels and how to elucidate the effect of irradiation times, and polymer and crosslinker concentrations on the hydrogel mechanical and structural properties. To this purpose, we combined complementary analysis including rheology, low field nuclear magnetic resonance spectroscopy (LFNMR) and small angle X-ray scattering (SAXS) to estimate the polymer mesh size of the gels prepared under different conditions. The fragile nature of most of the examined gels entailed an uncertain determination of polymer concentration in the gel according to the traditional approach based on gel weight measurements ${ }^{25,30-32}$ (both in the crosslinking conditions and in the swollen state). Accordingly, we developed an alternative method based on the LFNMR spectroscopy. We correlated the nuclear magnetic relaxation times of the gels with the ones of polymer solutions with defined concentration by means of a custom-made experimental model, developed starting from the theories of Chui ${ }^{33}$ and Scherer ${ }^{34}$ for the microstructural interpretation of LFNMR datasets.

The experimental results of network mesh size and crosslinking efficiency allowed a deeper understanding of the relationship between the mechanical behavior and the nanoscopic structure of the gels under consideration.

\section{Experimental}

\subsection{Materials}


Poly(vinylpyrrolidone) (PVP K90, $\mathrm{M}_{\mathrm{w}}=3.610^{5} \mathrm{Da}$ ), hydrogen peroxide $\left(\mathrm{H}_{2} \mathrm{O}_{2}\right)$ aqueous solutions (30\% wt.) and deuterium oxide (>99.9\% isotopic purity) were supplied by Sigma Aldrich. Water was deionized (DI). All the employed solvents were degassed before preparation of the solutions.

\subsection{Hydrogel preparation}

PVP solutions were prepared dissolving the polymeric powder in mixtures of DI water $(99.1 \% \mathrm{v} / \mathrm{v})$ and $\mathrm{H}_{2} \mathrm{O}_{2}(0.9 \% \mathrm{v} / \mathrm{v})$, with polymer concentration of 10, 20 and $30 \%$ wt. under stirring (250 rpm) at $50^{\circ} \mathrm{C}$ for $36 \mathrm{~h}$. Even at the highest concentration, PVP was perfectly dissolved, resulting in high viscous and transparent solutions. For the rheological and the LFNMR investigations, macroscopic specimens of the hydrogel were prepared. To investigate the influence of the crosslinker concentration on the structural properties, additional 30\% PVP solutions were prepared with double and triple amounts of $\mathrm{H}_{2} \mathrm{O}_{2}$ (referred as $30 \%+2 \mathrm{H}$ and $30 \%+3 \mathrm{H}$ ). From preliminary photo-crosslinking tests performed on layers of different thickness, it was found that gels thicker than 1 mm exhibit a macroscopically inhomogeneous structure with a softer texture at the bottom and a more elastic one on the top. Gels thinner than 1 mm were very fragile and unpractical to handle. As a consequence, a fixed thickness of $1 \mathrm{~mm}$ was used.

Solutions were cast into cylindrical moulds (1 mm in depth, $15 \mathrm{~mm}$ in diameter) and $0.5 \mathrm{~mm}$ thick glass discs were clamped onto the mold to prevent water evaporation during the UV exposure. It was measured that the glass absorbs a negligible amount of the radiation emitted by the UV lamps. Considering an emitted power density of $29 \mathrm{~mW} / \mathrm{cm}^{2}$, each sample was exposed to $67 \mathrm{~J}, 100 \mathrm{~J}$, $134 \mathrm{~J}$ corresponding to irradiations for 22, 33 and 44 min, respectively. After photo-crosslinking, gels were separated from the mould with a spatula and dipped in DI water under stirring to extract unreacted polymer and crosslinker (washing). The crosslinked polymer volume fraction after washing was $\phi_{0}$. Gels were, then, let to swell in water for $24 \mathrm{~h}$ and the polymer volume fraction went down to $\phi$. After swelling, the superficial water on the gels was dried.

In several cases, longer water soaking caused the breakage of the gel in smaller fragments probably due to a phenomenon of overswelling.

\subsection{Rheological measurements}

The rheological characterizations, carried out at $25^{\circ} \mathrm{C}$, were performed by means of a controlled stress rheometer Haake Rheo-Stress RS150 equipped with a thermostat F6/8. While a cone-plate device (C60/1 Ti: diameter $60 \mathrm{~mm}$, cone angle of $1^{\circ}$ ) was adopted for PVP solutions, a parallel plate device with serrated surfaces (PP20Ti: diameter $=20 \mathrm{~mm}$ ) was used for PVP gels. In the case of PP20Ti device, gap-setting optimization was undertaken according to a procedure described elsewhere ${ }^{35}$ (gap ranged between 1 and 2 $\mathrm{mm}$ ). In order to avoid water loss form the samples (gels or solutions) due to possible evaporation, the measurement device was kept inside a glass solvent trap at constant moisture level.

Solutions rheological characterization was led according to continuous test (flow curve test), implying the determination of solution viscosity versus the applied shear stress. Gels were studied by means of oscillatory tests. In particular, the linear viscoelastic region was assessed, at $1 \mathrm{~Hz}$, by stress sweep experiments. Frequency sweep tests were carried out in the frequency $(f)$ range $10-0.01 \mathrm{~Hz}$ at 
constant stress $5 \mathrm{~Pa}$ (well inside the linear viscoelastic range for all the studied gels). The generalized Maxwell model ${ }^{36}$ was used for the theoretical dependence of the elastic ( $\left.G^{\prime}\right)$ and viscous (G') moduli on angular frequency (or pulsation) $\omega=2 \pi f(f=$ stress frequency):

$$
\begin{aligned}
& G^{\prime}=G_{\mathrm{e}}+\sum_{i=1}^{n} G_{\mathrm{i}} \frac{\left(\lambda_{\mathrm{i}} \omega\right)^{2}}{1+\left(\lambda_{\mathrm{i}} \omega\right)^{2}} ; \quad G_{\mathrm{i}}=\eta_{\mathrm{i}} / \lambda_{\mathrm{i}}(1) \\
& G^{\prime \prime}=\sum_{i=1}^{n} G_{\mathrm{i}} \frac{\omega \lambda_{\mathrm{i}}}{1+\left(\lambda_{\mathrm{i}} \omega\right)^{2}}
\end{aligned}
$$

where $n$ is the number of Maxwell elements considered while $G_{\mathrm{i}}, \eta_{\mathrm{i}}$ and $\lambda_{\mathrm{i}}$ represent, respectively, the spring constant, the dashpot viscosity and the relaxation time of the $\mathrm{i}^{\text {th }}$ Maxwell element. $G_{\mathrm{e}}$ is the spring constant of the last Maxwell element which is supposed to be purely elastic ${ }^{36}$. The simultaneous fitting of Eqs. (1) and (2) to experimental G' and G', data was performed assuming that relaxation times $\left(\lambda_{\mathrm{i}}\right)$ were scaled by a factor of $10^{36}$. Hence, the parameters of the model are $2+n$ (i.e. $\lambda_{1}$, $G_{\text {e }}$ plus $G_{\mathrm{i}}$ ). Based on a statistical procedure ${ }^{37}, n$ was selected in order to minimize the product $\chi^{2}(1+n)$, where $\chi^{2}$ is the sum of the squared errors. According to the generalized Maxwell model, the elastic modulus varies between two limiting values: $G_{\min }\left(=G_{\mathrm{e}}\right.$; relaxed and deformed system after stress application) and $G_{\max }\left(=G_{\mathrm{e}}+\sum_{\mathrm{i}} G_{\mathrm{i}}\right)$ accounting for the structural characteristics of the unperturbed system ${ }^{38}$. As in the field of drug delivery the interest is focused on the properties of systems not exposed to mechanical perturbation, the shear modulus of gels $(G)$ is assumed coincident with $G_{\max }$. The application of Flory's theory ${ }^{39}$ enables the determination of the crosslink density $\rho_{\mathrm{x}}$ (defined as the moles of crosslinks between different polymeric chains per unit volume):

$$
\rho_{\mathrm{x}}=\frac{G}{R T}\left(\frac{\phi}{\phi_{0}}\right)^{\frac{2}{3}}
$$

where $R$ is the universal gas constant, $T$ is the absolute temperature while $\phi$ and $\phi_{0}$ are, respectively, the polymer volume fraction in the swollen state and in the reference state. Finally, the equivalent network theory ${ }^{40}$ allows evaluating the average network mesh size $\xi_{\mathrm{a}}$ :

$$
\xi_{\mathrm{a}}=\sqrt[3]{6 / \pi \rho_{\mathrm{x}} N_{\mathrm{A}}}
$$

where $N_{\mathrm{A}}$ is the Avogadro number.

\subsection{LFNMR spectroscopy measurements}

The characterization of hydrogel nanostructure was performed at $25^{\circ} \mathrm{C}$ according to low field ${ }^{1} \mathrm{H}$-NMR methodology, using Bruker Minispec mq20 (0.47 T, Germany). Two kinds of experimental tests were performed: a) determination of the water protons transverse relaxation time inside the hydrogels $\left(T_{2}\right)$ and b) determination of the water diffusion coefficient inside the hydrogel polymeric structure $\left(D_{\mathrm{g}}\right.$; also called water self-diffusion coefficient ${ }^{41}$. Water is the only mobile species as polymeric chains cannot diffuse, as the polymer fixed in the network does not diffuse).

$T_{2}$ measurements were performed according to the CPMG (Carr-Purcell-Meiboom-Gill) ${ }^{42}$ sequence with a $90-180^{\circ}$ pulse separation of $0.25 \mathrm{~ms}$ (number of scans: 4 ; delay: $5 \mathrm{~s}$ ). The $T_{2}$ discrete distribution was determined by fitting the time $(t)$ decay of the 
experimental signal $\left(I_{\mathrm{s}}\right)$, related to the extinction of the $x-y$ component of the magnetization vector $\left(M_{\mathrm{xy}}\right)$. The experimental $I_{\mathrm{s}}$ was fitted by its theoretical estimation $I(t)$ :

$$
I(t)=\sum_{i=1}^{m} A_{i} e^{-\frac{t}{T_{2 i}}}\left\langle\frac{1}{T_{2}}\right\rangle=\frac{\sum_{i=1}^{m} A_{i} T_{2 i}}{\sum_{i=1}^{m} A_{i}}
$$

where $t$ is time, $A_{\mathrm{i}}$ are the pre-exponential factors (dimensionless) proportional to the number of protons relaxing with the relaxation time $T_{2 \mathrm{i}}$ and $<1 / T_{2}>$ is the average value of the inverse relaxation time of protons. Again, $m$ was determined by minimizing the product $\chi^{2 *}(2 m)$, where $\chi^{2}$ is the sum of the squared errors and $2 m$ represents the number of fitting parameters of Eq.(5) ${ }^{37}$. The $T_{2}$ continuous distribution was determined according to the following equation:

$$
I(t)=\int_{T_{2 \min }}^{T_{2 \max }}\left(a\left(T_{2}\right) \exp \left(-\frac{t}{T_{2}}\right)\right) \mathrm{d} T_{2}
$$

where $T_{2 \max }$ is the relaxation time of free water at $25^{\circ} \mathrm{C}\left(T_{2 \mathrm{H} 2 \mathrm{O}}=3007 \mathrm{~ms}\right)$ and $T_{2 \min }(=0.1 \mathrm{~ms}$; lowest detectable experimental value of $T_{2}$ ) indicate, respectively, the lower and the upper values of the continuous $T_{2}$ distribution, $a\left(T_{2}\right)$ is the unknown amplitude of the spectral component at relaxation time $T_{2}$ and $e^{\left(-t / T_{2}\right)}$ represents the decay term. $a\left(T_{2}\right)$ determination required fitting of Eq.(6) to the experimental $I_{\mathrm{s}}$ data. Thus, Eq.(6) was discretized according to the criterion suggested by Whittal and MacKay ${ }^{43}$ :

$I(t)=\int_{T_{2}^{\text {min }}}^{T_{2}^{\max }} a\left(T_{2}\right) e^{\left(-t / T_{2}\right)} \mathrm{d} T_{2} \approx \sum_{i=1}^{N} a_{\mathrm{i}}\left(T_{2}^{\mathrm{i}}\right) e^{\left(-t / T_{\mathrm{2i}}\right)}\left(T_{2}^{\mathrm{i}+1}-T_{2}^{\mathrm{i}}\right)=\sum_{i=1}^{N} A_{i}\left(T_{2}^{\mathrm{i}}\right) e^{\left(-t / T_{2 \mathrm{i}}\right)}(7)$

where the range of the $T_{2}$ distribution $\left(T_{2 \min }-T_{2 \max }\right.$ ) was logarithmically divided into $\mathrm{N}=200$ segments (higher $N$ values have revealed to be unnecessary). Because of the noise disturbing the measure of $I_{s}$, a smoothed version $\left(\chi_{s}^{2}\right)$ was minimized:

$\chi_{\mathrm{s}}^{2}=\sum_{i=1}^{N}\left(\frac{I_{s}\left(t_{\mathrm{i}}\right)-I\left(t_{\mathrm{i}}\right)}{\sigma_{\mathrm{i}}}\right)^{2}+\mu \sum_{i=1}^{N-2}\left|A_{\mathrm{i}+2}-2 A_{\mathrm{i}+1}+A_{\mathrm{i}}\right|^{2}$

where $\sigma_{\mathrm{i}}$ is the $\mathrm{i}^{\text {th }}$ data point standard deviation, $\mu$ the is weight of the smoothing term (second sum in Eq.(8)) proposed by Provencher ${ }^{44}$. For the determination of $\mu$, the method adopted by Wang ${ }^{45}$ was chosen. The selected $\mu$ value $(=261)$ is that occurring just after the heel (slope variation) of the function $\operatorname{Ln}\left(\chi_{\mathrm{s}}\right)$ vs $\operatorname{Ln}(\mu)$.

The results of the relaxation experiments were also used for the estimation of the mesh size distribution of the polymer networks. The theory of Scherer ${ }^{34}$ states that:

$$
R_{\mathrm{h}}=\frac{\xi_{\mathrm{a}}^{2}}{R_{\mathrm{f}}} \frac{C_{0}(1-\phi)}{\left(C_{1}-1.5 C_{2} R_{\mathrm{f}} / \xi_{\mathrm{a}}\right)}
$$

where $R_{\mathrm{f}}$ is the radius of the polymeric chain, $\xi_{\mathrm{a}}$ is the average network mesh size, $\phi$ is the polymer volume fraction, $C_{0}, C_{1}$ and $C_{2}$ are geometrical constants for a cubic mesh, equal to $1,3 \pi$ and $8 \sqrt{2}$, respectively. $R_{\mathrm{h}}$ represents the hydraulic radius of the cubic mesh, i.e. two times the ratio between the cubic cell volume and the surface area of the polymeric chains of the cubic cell. In addition, Scherer demonstrated that a good approximation of eq.(9) is given by: 


$$
R_{\mathrm{h}}=R_{\mathrm{f}}\left(\frac{1-0.58 \phi}{\phi}\right)
$$

Here we assume that $R_{\mathrm{f}} / \xi_{\mathrm{a}} \approx 0$ and eq.(9) becomes:

$$
R_{\mathrm{h}} \approx \frac{\xi_{\mathrm{a}}^{2}}{R_{\mathrm{f}} C_{1}} C_{0}(1-\phi)
$$

Combination of eq.(10) and (11) yields to a simple and direct relation between $R_{\mathrm{f}}$ and $\xi_{\mathrm{a}}$ :

$$
\xi_{\mathrm{a}}=R_{\mathrm{f}} \sqrt{\frac{(1-0.58 \phi)}{(1-\phi) \phi} \frac{C_{1}}{C_{0}}}
$$

It can be numerically demonstrated that, in the range $0<\phi<0.1$, the error associated with the use of eq.(12) in place of eq.(9) for the estimation of $\xi_{\mathrm{a}} / R_{\mathrm{f}}$ is always lower than $10 \%{ }^{46}$

The relation between $\xi_{\mathrm{a}}$ and $<1 / T_{2}>$ is provided by the "Fiber-Cell" theory"

$$
\left\langle\frac{1}{T_{2}}\right\rangle=\frac{1}{T_{2 \mathrm{H}_{2} \mathrm{O}}}+\frac{2}{f} \frac{\langle\mathcal{M}\rangle}{\xi_{\mathrm{a}}} \quad f=\sqrt{\frac{(1-0.58 \phi)(1-\phi)}{\phi} \frac{C_{0}}{C_{1}}}
$$

where $T_{2 \mathrm{H} 2 \mathrm{O}}$ is the relaxation time of the bulk water protons (i.e. protons of the free water, whose relaxation is not affected by the presence of the polymeric chains) and $\langle\mathcal{M}\rangle$ is an empirical parameter, called relaxivity, accounting for the effect of polymer chains surface on proton relaxations. While Eq.(13) holds, on average, for all the polymeric class of network meshes, similar expressions can be written for polymeric network meshes of different dimensions:

$$
\frac{1}{T_{2 \mathrm{i}}}=\frac{1}{T_{2 \mathrm{H}_{2} \mathrm{O}}}+\frac{2}{f} \frac{\langle\mathcal{M}\rangle}{\xi_{\mathrm{i}}}
$$

where $T_{2 \mathrm{i}}$ is the relaxation time of the water molecules protons trapped in polymeric meshes of size $\xi_{\mathrm{i}}\left(\langle\mathcal{M}\rangle\right.$ is independent from $\xi_{\mathrm{j}}$ ). Eq.(13) and (14) hold in fast-diffusion regime ${ }^{33}$,i.e when the mobility of the water molecules, expressed by their self-diffusion coefficient $D_{\mathrm{g}}$, is high compared to the rate of magnetization loss, identifiable with $\langle\mathcal{M}\rangle R_{\mathrm{c}}\left(\langle\mathcal{M}\rangle R_{\mathrm{c}} / D_{\mathrm{g}}<<1\right)$. $R_{\mathrm{c}}$ indicates the distance from the polymer chain axis where the effect of polymeric chains on water protons relaxation becomes negligible and can be expressed by:

$$
R_{\mathrm{c}}=\frac{R_{\mathrm{f}}}{\sqrt{\phi}}
$$

When the assumptions of fast-diffusion regime do not hold, the relaxation of water protons trapped in a mesh of diameter $\xi_{\mathrm{i}}$ needs to be described by, in theory, an infinite number of relaxation times $\left(T_{2 i j}\right)$. Accordingly, one single relaxation time $\left(T_{2 \mathrm{i}}\right)$ is no longer sufficient in eq.(5), (13) and (14) (this occurs when $\xi_{\mathrm{i}}$ greater than $1 \mu \mathrm{m}$, i.e, like for microporous systems). 
With the value of $\langle\mathcal{M}\rangle \xi_{\mathrm{i}}$ is evaluated by eq.(14) for each class of the network meshes. In addition, by knowing $\langle\mathcal{M}\rangle$ and the continuous distribution of $T_{2}, a\left(T_{2}\right)$, (see Eq.(7)), the continuous distribution of $\xi, a(\xi)$, is determined. Indeed, the following relations hold:

$$
\begin{aligned}
& \xi=\frac{2\langle\mathcal{M}\rangle}{f} /\left(\frac{1}{T_{2}}-\frac{1}{T_{2 \mathrm{H}_{2} \mathrm{O}}}\right) \\
& a(\xi)=\frac{d T_{2}}{d \xi} a\left(T_{2}\right)=\frac{f}{2\langle\mathcal{M}\rangle}\left(\frac{T_{2 \mathrm{H}_{2} \mathrm{O}}-T_{2}}{T_{2 \mathrm{H}_{2} \mathrm{O}}}\right)^{2} a\left(T_{2}\right)
\end{aligned}
$$

In addition, for a more clear description the probability of finding a mesh of size $\xi$ inside the polymeric network $P(\xi)$ is estimated as follows:

$$
P(\xi)=\frac{a(\xi) d \xi}{\int_{\xi_{\min }}^{\xi_{\max }} a(\xi) d \xi}=\frac{A(\xi)}{\int_{\xi_{\min }}^{\xi_{\max }} a(\xi) d \xi}
$$

In order to gain further information about polymeric network structure, Pulsed Gradient Spin Echo (PGSE) measurements were performed. The applied sequence consisted of the classical CPMG sequence with two equal gradient pulses (of length $\delta=0.5 \mathrm{~ms}$ ) occurring at $\mathrm{x}_{1}=1 \mathrm{~ms}$ and $\mathrm{x}_{2}=1 \mathrm{~ms}$ after the 90 and $180^{\circ}$ pulses, respectively. The time separation, indicated by $\Delta\left(\approx \tau-\mathrm{x}_{1}-\delta+\mathrm{x}_{2}\right)$, is related to the water molecule diffusion time $t_{\mathrm{d}}$ according to $t_{\mathrm{d}} \approx \Delta-\delta / 3$. After a proper calibration procedure, based on the knowledge of the free water self-diffusion coefficient $\left(D_{\mathrm{H} 2 \mathrm{O}}\right)^{47}$ it is possible to measure the average water self-diffusion coefficient inside the hydrogel $\left(D_{\mathrm{g}}\right)$. Interestingly, for sufficiently long $t_{\mathrm{d}}$ (this happens when $D_{\mathrm{g}}$ is no longer dependent on $t_{\mathrm{d}}$; for small $t_{\mathrm{d}}$, $D_{\mathrm{g}}$ decreases with increasing $\left.t_{\mathrm{d}}\right)$ the ratio between $D_{\mathrm{H} 20}$ and $D_{\mathrm{g}}$ identifies with the polymeric network tortuosity $(\alpha)^{48}$.

\section{$2.5 R_{\mathrm{f}}$ determination}

$R_{\mathrm{f}}(0.91 \pm 0.10 \mathrm{~nm})$ was evaluated according to molecular mechanics (MM)/dynamics (MD) techniques assuming prototypical linear and atattic short monomer-PVP chains (8 units). Ten different chains were modelled applying the isomeric-rotational method developed by Theodorou and Suter ${ }^{49}$ at $25^{\circ} \mathrm{C}$. Each chain was geometrically optimized and equilibrated following a protocol based on molecular mechanic/heating cycles coupled to molecular dynamics ${ }^{50-52}$. Then, the models were placed in a tridimensional periodic simulation box with explicit solvent (TIP3P water model ${ }^{53}$ ). After minimization, the hydrated PVP chains were subjected to 1 ns equilibration run in the isobaric-isothermal ensemble (NPT MD) at $25^{\circ} \mathrm{C}$ and 1 bar (temperature and pressure were maintained using a Berendsen thermostat and barostat ${ }^{54}$ ). Then, 2 ns production run was carried out at constant volume and temperature (NVT MD). The time step was $1 \mathrm{fs}$ for all simulations. For the long-range electrostatic interactions, particle mesh Ewald ${ }^{55}$ was used. A cutoff radius of $12.5 \AA$ A was assumed for all interactions. For cross-section area analysis, 100 snapshots were saved during the last 500 ps of the NVT MD production period described above, and averaged over the 10 chains models. All simulations were performed by means of Materials Studio software v. 5.5 (Accelrys, San Diego, CA) and employing COMPASS ${ }^{56}$ force field. 


\subsection{SAXS measurements}

A collimated beam, with a wave-length $\lambda=0.1542 \mathrm{~nm}$, was irradiated through a vacuum sealed cell containing the gel sample. The scattered field was then detected with a 2D camera, which allowed the evaluation of the diffracted intensity down to $q=0.1 \mathrm{~nm}^{-1}$. While $q$ is the scattering vector defined as $q=(4 \pi)^{*} \sin (\theta) / \lambda$., $\theta$ is the angle between the incident and the scattered field. Intensities were collected at room temperature for 30 minutes. Raw data were corrected for dark current and background and then normalized with respect to the primary beam intensity. A further correction for both Porod constant and the de-smearing effect was applied and the results were plotted as $I q^{2} v s q$.

\section{Results and discussion}

\subsection{Polymer volume fraction determination}

Eqs.(3), (4) and (12), aimed at the evaluation of the PVP network mesh size, require knowledge of the polymer volume fraction in the reference $\left(\phi_{0}\right)$ and in the swollen $(\phi)$ conditions. However, the preparation procedure implied a washing after crosslinking, which made the estimation of $\phi_{0}$ as well as the PVP volume fraction in the swollen condition $(\phi)$ very difficult. For this purpose, a semiempirical approach, based on LF-NMR, was designed in order to estimate $\phi_{0}$ and $\phi$ for the different hydrogels studied. This approach is based on the experimental evidence that the average relaxation time $\left(T_{2 \mathrm{~g}}\right)$ of a polymeric hydrogel is always lower than the one of the aqueous solutions $\left(T_{2 \mathrm{~s}}\right)$ with equal polymer volume fraction. Thus, the hydrogel and the polymeric solution differ only for the different arrangement of the polymeric chains due to the presence of the crosslinks occurring in the hydrogel. It can be assumed that while in a solution the polymeric chains are in a curled configuration with intramolecular and possibly in clusters, in the case of a gel the crosslinks constrain the chains in a three-dimensional network, where the polymer configuration is less curled and the surface accessible to water is higher. Based on these assumptions, the curled state implies a reduced surface area $(S)$ of the polymer/water interface that is the key point for the determination of the water protons relaxation time. The original Chui theory ${ }^{33}$ states:

$$
\left\langle\frac{1}{T_{2}}\right\rangle=\frac{1}{T_{2 \mathrm{H}_{2} \mathrm{O}}}+\frac{2\langle\mathcal{M}\rangle}{R_{\mathrm{h}}} \quad R_{\mathrm{h}}=\frac{2 V}{S}
$$

where $R_{\mathrm{h}}$ is the hydrodynamic radius while $V$ is a volume proportional to the solvent (water in our case) volume fraction (1- $\left.v_{\mathrm{p}}\right)$ in the gel. While $V$ weakly depends on the conformation assumed by the polymeric $\operatorname{chain}^{57}, S$ is strongly dependent on the chains conformation and it will be higher in the case of a linear configuration while it will decrease in the case of curled chains. Being $v_{\mathrm{p}}$ (polymer volume fraction in the gel) the same, $\mathrm{R}_{\mathrm{h}}$ in a gel network will be lower than in a solution. As a consequence, from eq.(19) it follows that $\left\langle 1 / T_{2}>_{\text {gel }}\right.$ is bigger than $\left\langle 1 / T_{2}\right\rangle_{\text {sol }}$ being $\langle\mathcal{M}\rangle$ and free $T_{2 \mathrm{H} 2 \mathrm{O}}$ the same for both systems (hydrogel and solution). Thus, $T_{2 \mathrm{gel}}$ $<T_{2 \text { sol. }}$. As for a hydrogel, eq.(14) can be re-written as:

$$
\left\langle\frac{1}{T_{2}}\right\rangle_{g e l}=\frac{1}{T_{2 \mathrm{H}_{2} \mathrm{O}}}+\frac{2\langle\mathcal{M}\rangle}{R_{\mathrm{f}}} \frac{v_{\mathrm{p}}}{1-0.58 v_{\mathrm{p}}}
$$

We assumed that, for a solution, the following relation holds: 


$$
\left\langle\frac{1}{T_{2}}\right\rangle_{\text {sol }}=\frac{1}{T_{2 \mathrm{H}_{2} \mathrm{O}}}+\frac{2\langle\mathcal{M}\rangle}{R_{\mathrm{f}}} \frac{v_{\mathrm{p}}^{\mathrm{n}}}{1-0.58 v_{\mathrm{p}}^{\mathrm{n}}}
$$

where $n$ is an empirical fitting parameter that is equal to 1 in the case of hydrogels and it has to be greater than 1 in case of solutions in order to yield $T_{2 \text { gel }}<T_{2 \text { sol }}$. Accordingly, eq.(21) was fitted to the experimental relaxation data referring to PVP aqueous solutions characterized by different, and experimentally known, polymer volume fractions $\left(v_{p}\right)$ as shown in Figure 1 (filled circles). Eq.(21) fitting to experimental data was statistically reliable, as proved by the $F$-test $(F(1,16,0.95)<235)$, and fitting parameters resulted $n$ $=(1.9 \pm 0.3)$ and $\langle\mathcal{M}\rangle=(7.7 \pm 2.9) 10^{-3} \mathrm{~nm} / \mathrm{ms}$. The knowledge of $\langle\mathcal{M}\rangle$ was used to determine $v_{\mathrm{p}}$ from eq.(20) as $\left\langle 1 / T_{2}\right\rangle_{\text {gel }}$ is known from experimental measurements. For the different examined systems, Table 1 shows the values of $\phi_{0}$ and $\phi$ obtained with this approach. It can be seen that the washing/swelling processes implies a considerable sol fraction, i.e. uncrosslinked portion of the polymer, of the system considered.

\subsection{Rheological characterization}

Stress sweep tests revealed (data not shown) that, for all the studied gels, the linear viscoelastic range holds for stresses above the value adopted for the frequency sweep tests $(\tau=5 \mathrm{~Pa}$ ). In addition, Figure 2 reports the mechanical spectra referring to systems 10 -B, 20-B and 30-B in the swollen state. As, for all the three systems, the storage ( $\left.G^{\prime}\right)$ and the loss (G') moduli are substantially independent from the angular frequency $\omega(=2 \pi f)$ and $G$ ' is definitely prevalent on $G^{\prime \prime}$, they can be defined as gels ${ }^{58}$. However, the typical gel characteristics (for instance the ratio $G$ ' $G$ '’ $>10$ ), are much more pronounced passing from 10-B to 20-B than from 20-B to 30-B. Notably, in the series 10-B to 30-B, while G', is more or less unchanged, G' undergoes an increase of one order of magnitude. It is worth notice that, differently from what reported by D’Errico et al. ${ }^{59}$, while $G^{\prime}$ increases with the concentration of the originating polymeric solution $(10,20,30 \% \mathrm{wt})$, the polymer volume fraction $\phi$ corresponding to these systems is almost the same (see Table 1). Thus, the increase of the polymer concentration of the initial polymer solution does not entail an increase of the gel fraction: reasonably, the number of polymeric chains involved in the crosslinking reaction remains constant whilst an increase of initial PVP concentration or of irradiation time produces more crosslink points among a stable number of polymeric chains. Table 2, showing the crosslink density, $\rho_{\mathrm{x}}$, regarding gels 10-B, 20-B and 30-B, seems to confirm this hypothesis. Three Maxwell elements plus a purely elastic element $\left(G_{\mathrm{e}}\right)$ (see eq.(1) and (2)) are needed for a statistically reliable fitting of the frequency sweep data shown in Figure 2. The data fitting reveals also that the average mesh size ( $\xi_{\text {aRHEO }}$ ) of these gels ranges between 65 and $48 \mathrm{~nm}$, where 10 -B gel exhibits the highest $\xi_{\text {aRHEO. }}$

The analysis of gels 10-A, 10-C, 20-A, 20-C, 30-A and 30-C led to conclusions similar to those reported for the above discussed 10B, 20-B and 30-B gels (data not shown). Figure 3 sums up the results of the characterization of gels 10-ABC, 20-ABC and 30-ABC by showing the trend of the shear modulus $G$. It is evident that $G$ increases both with the crosslinking time (horizontal axis) and with the increase of the polymer volume fraction of the originating solution $(10 \%, 20 \%, 30 \%)$. It is interesting to notice that, for the "C” gels, the increase of $G$, in the series 10-20-30, is more pronounced than those of the “A” and "B” gels (see Figure 3). 
Figure 4 shows the results of the frequency sweep tests related to systems characterized by an increasing initial concentration of crosslinker $\left(\mathrm{H}_{2} \mathrm{O}_{2} ; 1 \mathrm{H}, 2 \mathrm{H}, 3 \mathrm{H}\right)$. In particular, the attention is focussed on 30-B, 30-B-2H and 30-B-3H gels. These samples clearly belong to the viscoelastic class of gels as $G$ ' independent on pulsation $\omega, G$ ', is weakly dependent and $G$ ' is neatly prevailing on $G$ '. Substantially, the increase of the crosslinker amount reflects into an increase of $G$ ' whereas $G$ ', is almost constant. The increase of $G$ ' with the $\mathrm{H}_{2} \mathrm{O}_{2}$ concentration is more gradual than in the case of the increase of the originating polymeric solution (see Figure 2). Two/three Maxwell elements plus a purely elastic element $\left(G_{e}\right)$ (see eq.(1) and (2)) are needed for a statistically reliable fitting of the frequency sweep data shown in Figure 4 (see Table 3).

From Table 3 it can be noticed that the increase of the crosslinker amount $\left(\mathrm{H}_{2} \mathrm{O}_{2}\right)$ corresponds to an increase of the crosslink density $\left(\rho_{\mathrm{x}}\right)$ and a consequent decrease of the average mesh size $\left(\xi_{\mathrm{aRHEO}}\right)$. Qualitatively similar conclusions can be drawn for the gels 30-A, 30-A-2H, 30-A-3H, 30-C, 30-C-2H and 30-C-3H.

Figure 5 shows the trend of the shear modulus $G$ with the crosslinker amount of the $30 \%$ PVP gels with different $\mathrm{H}_{2} \mathrm{O}_{2}$ concentration. For all these gels, $G$ increases with the crosslinker content and this trend is maximised for the 30-C series.

\subsection{LF-NMR and SAXS characterization}

LF-NMR analysis revealed that all the studied gels are characterized by only one relaxation time $\left(T_{2}\right)$ (see Table 1). This means that all the hydrogels are homogeneous systems, i.e. all the water molecules interact with the polymeric chains in the same manner. Consequently, in the light of the Scherer's theory ${ }^{34}$, these gels are characterized by meshes of similar dimensions. On the contrary, the highly heterogeneous gels, such as those prepared from alginate, up to four relaxation times can be found ${ }^{47}$. Relying on measurements of $T_{2}$ and on eq.(12), it is possible estimating the average mesh size ( $\xi_{\text {aNMR }}$ ) of our hydrogels as shown in Table 4. It can be noticed that $\xi_{\mathrm{aNMR}}$ is almost constant and spans from $31 \mathrm{~nm}$ to $44 \mathrm{~nm}$. These high values of $\xi_{\mathrm{aNMR}}$ are comparable with the evaluation of the water self-diffusion coefficient $\left(D_{\mathrm{g}}\right)$ in the gels. Indeed, for all gels, $D_{\mathrm{g}}$ is very close to the value of the free water self-diffusion coefficient at $25^{\circ} \mathrm{C}\left(2.3^{*} 10^{-9} \mathrm{~m}^{2} / \mathrm{s}{ }^{41}\right)$. This means that the hindering action exerted by polymeric chains on water molecules diffusion is very weak as occurs with gels characterized by wide meshes. SAXS analysis reveals that, in the investigated range of scattering vector $q$, experimental data do not display any significant order length. Indeed, the features, reported in Figure 6 (referring, for example, to gel 20-B), are more typical of a semi-flexible random coil.

In order to interpret the SAXS datasets, the worm like chain (WLC) model ${ }^{60}$ was adopted. In this model, the polymeric chain is characterized by two characteristic lengths: the contour length $(L)$ and the persistence length $\left(l_{\mathrm{p}}\right)$. In the limit of $L>>l_{\mathrm{p}}$, the following relationship exists between the scattering intensity $I(q)$ and the scattering vector $q$ :

$$
\frac{\mathrm{I}(\mathrm{q})}{\mathrm{I}(0)}=2 \mathrm{u}^{-2}[\exp \{-\mathrm{u}\}-1+\mathrm{u}]+\frac{\mathrm{l}_{\mathrm{p}}}{\mathrm{L}}\left[\frac{4}{15}+\frac{7}{15 \mathrm{u}}-\left(\frac{11}{15}+\frac{7}{15 \mathrm{u}}\right) \exp \{-\mathrm{u}\}\right]
$$

where 


$$
\mathrm{u}=\frac{\mathrm{Ll}_{\mathrm{p}} \mathrm{q}^{2}}{6}
$$

Eq.(22) best fitting to experimental data shown in Figure 6 yields to the following values of the two lengths: $L=547 \mathrm{~nm}, l_{\mathrm{p}}=2.13$ $\mathrm{nm}$. These values are compatible with the average mesh size found. Indeed, the value of $L$ implies that, on average, each polymeric chain is involved in about $10-20$ meshes. In addition, the persistence length $l_{\mathrm{p}}$ (rigid chain portion) seems compatible with meshes spanning in the range $30-44 \mathrm{~nm}$ (see Table 3 and 4).

Figure 7 reports the comparison between the average mesh diameter evaluated by means of rheology and LF-NMR for the gels 10 ABC, 20-ABC and 30-ABC. In order to perform a more exhaustive comparison, the horizontal axis reports the shear modulus ( $G$ ) competing to each gels. It can be observed that when $G$ exceeds about $140 \mathrm{~Pa}$, both methods lead to similar values. However, for smaller $G$, rheology gives to a larger estimation of the average mesh size. This fact could be explained by the presence of dangling chains that, being bound to one end of the network, are elastically inactive and, thus, irrelevant for the determination of $G$ and $\xi$ according to the rheological approach (see eq.(3)) ${ }^{61}$. On the contrary, for the LF-NMR approach (see eq.(12)), both elastically active and inactive chains are important. As the number of dangling chains can be roughly connected to the viscous properties of the gel ( $\left.G^{\prime \prime}\right)$, it is reasonable to say that dangling chains have a smaller and smaller effect when $G$ increases. In our case, this $G$ threshold appears to be around $140 \mathrm{~Pa}$.

Figure 8, reporting the comparison between the average mesh diameter evaluated by means of rheology and LF-NMR for the gels 30A-2H-3H, 30-B-2H-3H and 30-C-2H-3H, substantially confirms the above discussed experimental evidences. Indeed, for $G>100$ Pa, the rheological and the LF-NMR approach yield to similar estimation of the average mesh size.

Finally, Figure 9 reports the continuous mesh distribution referring to gel 20-A (chosen as a representative example of all other gels) determined according to the LF-NMR approach (see eq.(18)). It can be seen that the most probable mesh size is around $40 \mathrm{~nm}$ while the distribution spans from about $25 \mathrm{~nm}$ up to $120 \mathrm{~nm}$.

\section{Conclusions}

PVP hydrogels have been prepared by photo-reticulation of the polymer aqueous solutions. Chain crosslinking is determined by on recombination of macroradicals produced by UV irradiation of $\mathrm{H}_{2} \mathrm{O}_{2}$ which is used as photo-initiator. The effects of irradiation dose and of the polymer and photo-initiator concentration were elucidated by means of a combined use of two experimental techniques: rheology and low field NMR. The comparison of the results, leading to different estimation of the network mesh size, revealed the presence of a complex structure of the hydrogel networks, comprehending more elastically active chains, and an elastically inactive/viscous portion composed by dangling chains, experimentally "invisible” to the rheological measurements. This interpretation was confirmed both by the measurement of 
the water self-diffusion coefficient and the by SAXS in all the samples under study. Accordingly, the two methods converge to similar mesh size values in the stronger hydrogel where the elastic response prevails over the viscous one. These encouraging results suggest that this technique can potentially find further applications on other types of hydrogel networks.

\section{Acknowledgements}

This project was part of IDUN centre of excellence funded by the Danish National Research Foundation (DNRF 122) and the Villum Foundation (grant no. 9301). This work was supported by the Italian Ministry of Education (PRIN 2010-11 (20109PLMH2)). The authors thank Stephania Baldursdottir (University of Copenhagen), Thomas Rades (University of Copenhagen), Anette Müllertz (University of Copenhagen) and Sabrina Pricl (University of Trieste) for the interesting and fruitful discussions.

\section{Notes and references}

${ }^{a}$ Department of Micro- and Nanotechnology, Technical University of Denmark (DTU), Kongens Lyngby, Ørsteds Plads Bygning $345 \varnothing, 2800$ Kgs, Denmark.

${ }^{b}$ Department of Life Sciences, Cattinara Hospital, University of Trieste, Strada di Fiume 447, 34100 Trieste, Trieste, I-34127.

${ }^{c}$ Department of Engineering and Architecture, University of Trieste, via Valerio 6, Trieste, I-34127.

${ }^{d}$ Department of Chemical and Biochemical Engineering, Technical University of Denmark (DTU), Kongens Lyngby, Søltofts Plads 227, 2800 Kgs., Denmark.

${ }^{e}$ National Research Council (CNR), Institute of Polymer Composites and Biomaterials, P.le Enrico Fermi 1, 80055 Portici Napoli Italy. ste

1 Peppas, N. A., Bures P., Leobandung, W., and Ichikawa, H., Eur. J. Pharm. Biopharm., 50, 27-46 (2000).

2 Ishihara, M., Nakanishi, K., Ono, K., Sato, M., Kikuchi, M., Saito, Y., Yura, H., Matsui, T., Hattori, H., Uenoyama, M. and Kurita, A., Biomaterials, 23, 833-840 (2002). Kirker, K. R., Luo, Y., Nielson, J. H., Shelb,y J. and Prestwich, G. D., Biomaterials, 23, 3661-3671 (2002). Jeong, B., Bae, Y. H., and Kim, S. W., J. Control. Release, 63, 155-163 (2000). Yu, L. and Ding, J., Chem. Soc. Rev., 37, 1473-81 (2008). 

57-64 (2008).

Mahoney, M. J., and Anseth, K. S., Biomaterials, 27, 2265-74 (2006).

Nguyen, K. T., and West, J. L., Biomaterials, 23, 4307-4314 (2002).

Am Ende, M. T., and Peppas, N. A., J. Control. Release, 48, 47-56 (1997).

Brazel, C. S. and Peppas, N. A., Eur. J. Pharm. Biopharm., 49, 47-58 (2000).

Grassi, M., Lapasin, R., Coviello, T., Matricardi, P., Di Meo, C., and Alhaique, F., Carbohydr. Polym., 78, 377-383 (2009).

Turco, G., Donati, I., Grassi, M., Marchioli, G., Lapasin, R., and Paoletti, S., Biomacromolecules, 12, 1272-82 (2011).

Ajji, Z., Othman, I., and Rosiak, J. M., Nucl. Instruments Methods Phys. Res. Sect. B Beam Interact. with Mater. Atoms, 229, 375-380 (2005).

Fernandes, R., and Gracias, D. H., Adv. Drug Deliv. Rev., 64, 1579-89 (2012).

Chirra, H. D., and Desai, T. A., Small, 8, 3839-46 (2012).

Fox, C. B., Chirra, H. D., and Desai, T. A., Curr. Pharm. Biotechnol., 15, 673-83 (2014).

Marizza, P., Keller, S. S., and Boisen A., Microelectron. Eng., 111, 391-395 (2013).

Marizza, P., Keller, S. S., Müllertz, A., and Boisen, A., J. Control. Release, 173, 1-9 (2014).

Casadei, M. A., Cerreto, F., Cesa, S., Giannuzzo, M., Feeney, M., Marianecci, C. and Paolicelli, P., Int. J. Pharm., 325, 140-6 (2006).

Pitarresi, G., Casadei, M. A., Mandracchia, D., Paolicelli, P., Palumbo, F. S., and Giammona, G., J. Control. Release, 119, 328-38 (2007).

Tripodo, G., Pitarresi, G., Palumbo, F. S., Craparo, E. F., and Giammona, G., Macromol. Biosci., 5, 1074-84 (2005).

Decker, C., Macromol. Rapid Commun., 23, 1067-1093 (2002).

Zhu, X., Lu, P., Chen, W., and Dong, J., Polymer (Guildf)., 51, 3054-3063 (2010). 
Haaf, F., Sanner, A., and Straub, F., Polym. J., 17, 143-152 (1985).

Rosiak, J., Olejniczak, J. and Pȩkala, W., Int. J. Radiat. Appl. Instrumentation. Part C. Radiat. Phys. Chem., 36, 747-755 (1990)

Lopérgolo, L. C., Lugão, A. B., and Catalani, L. H., Polymer (Guildf)., 44, 6217-6222 (2003).

Fechine, G. J. M., Barros, J. A. G., and Catalani, L. H, Polymer (Guildf)., 45, 4705-4709 (2004).

D’Errico, G., De Lellis, M., Mangiapia, G., Tedeschi, A., Ortona, O., Fusco, S., Borzacchiello, A. and Ambrosio, L., Biomacromolecules, 9, 231-40 (2008).

Chui, M. M., Phillips, R. J. and McCarthy, M. J., J. Colloid Interface Sci., 174, 336-344 (1995).

Scherer, G. W., J. Sol-Gel Sci. Technol., 1, 285-291 (1994).

Kuijpers, A. J., M., Engbers, G. H., Feijen, J., De Smedt, S. C., Meyvis, T. K. L., Demeester, J., Krijgsveld J., Zaat S. A. J. and Dankert J., Macromolecules, 32, 3325-3333 (1999).

Lapasin, R., and Pricl, S., in Rheology of industrial polysaccharides: theory and applications, pp. 250-494 (1995).

Draper N. R. and Smith H., J. R. Stat. Soc., 48, 140-141 (1999).

Pasut, E., Toffanin, R., Voinovich, D., Pedersini, C., Murano, E., and Grassi, M., J. Biomed. Mater. Res. A, 87, 808-18 (2008).

Flory, P. J., Principles of polymer chemistry, Cornell University Press, (1953).

Schurz, J., Prog. Polym. Sci., 16, 1-53 (1991).

Holz, M., , Heil S. R., and Sacco, A., Phys. Chem. Chem. Phys., 2, 4740-4742 (2000).

Meiboom, S., and Gill, D., Rev. Sci. Instrum., 29, 688 (1958).

Whittall K. P. and MacKay A. L., J. Magn. Reson., 84, 134-152 (1989).

Provencher, S. W., Comput. Phys. Commun., 27, 213-227 (1982).

Wang, X., and Ni, Q., J. Orthop. Res., 21, 312-9 (2003).

Coviello, T., Matricardi, P., Alhaique, F., Farra, R., Tesei, G., Fiorentino, S., Asaro F., Milcovich G., and Grassi. M., Express Polymer Letters, 7(9) 733-746 (2013). 

37 (2014). (2009).

Prathab, B., and Aminabhavi, T. M., Langmuir, 23, 5439-44 (2007).

Mahabadi, H. K., and Rudin, A., Polym. J., 11, 123-131 (1979).

Winter, H. H., J. Rheol. (N. Y. N. Y)., 30, 367 (1986).

D’Errico, G., De Lellis, M., Mangiapia, G., Tedeschi, A., Ortona, O., Fusco, S., Borzacchiello, A., and Ambrosio, L., Biomacromolecules, 9, 231-240 (2008). 